\title{
Mechanical Properties and Fracture Patterns of Graphene (Graphitic) Nanowiggles
}

\author{
Rafael A. Bizao ${ }^{1,2}$, Tiago Botari ${ }^{1}$, Eric Perim ${ }^{1,3}$, Nicola M. Pugno ${ }^{2,4,5}$, and \\ Douglas S. Galvao, ${ }^{1, *}$ \\ ${ }^{1}$ Instituto de Física Gleb Wataghin, Universidade Estadual de Campinas, 13083-970, Campinas, \\ SP, Brazil. \\ ${ }^{2}$ Department of Civil, Environmental and Mechanical Engineering, Laboratory of Bio-Inspired \\ and Graphene Nanomechanics, University of Trento, via Mesiano, 77, 38123 Trento, Italy. \\ ${ }^{3}$ Department of Mechanical Engineering and Materials Science, Duke University, Durham, NC \\ 27708, USA. \\ ${ }^{4}$ Italian Space Agency, Via del Politecnico snc, 00133 Rome, Italy. \\ ${ }^{5}$ School of Engineering and Materials Science, Queen Mary University of London, Mile End \\ Road, London E1 4NS, United Kingdom. \\ *galvao@ifi.unicamp.br
}

\begin{abstract}
Graphene nanowiggles (GNW) are graphene-based nanostructures obtained by making alternated regular cuts in pristine graphene nanoribbons. GNW were recently synthesized and it was demonstrated that they exhibit tunable electronic and magnetic properties by just varying their shape. Here, we have investigated the mechanical properties and fracture patterns of a large number of GNW of different shapes and sizes using fully atomistic reactive molecular dynamics simulations. Our results show that the GNW mechanical properties are strongly dependent on its shape and size and, as a general trend narrow sheets have larger ultimate strength and Young's modulus than wide ones. The estimated Young's modulus values were found to be in a range of $\approx 100-1000 \mathrm{GPa}$ and the ultimate strength in a range of $\approx 20-110$ GPa, depending on GNW shape. Also, super-ductile behaviour under strain was observed for some structures.
\end{abstract}

\section{Introduction}

Graphene is a carbon allotrope obtained by arranging carbon atoms on twodimensional (2D) honeycomb lattice. The advent of graphene [18, 29] created a revolution in materials science, due to its unique and exceptional electronic 
and mechanical properties. Because of these properties, graphene has great potential for applications in different fields, such as energy storage [28, 37], solar cells [34] and nanoelectronics [36]. However, in its pristine form, graphene is a zero band gap semiconductor, which poses limitations to its use in applications such as digital transistors. There are several ways to open graphene band gap, including chemical functionalization [6], application of mechanical stress [41] or by topological structural changes, notably the synthesis of narrow strips called graphene nanoribbons (GNR) [38. GNR can be defined as finite graphene segments with large aspect ratio. Their electronic properties have been extensively studied [2, 22] and shown to be directly related to electron confinement arising from constraints due to finite boundaries. In this way, as GNR becomes narrower the band gap increases, lowering the conductance [22].

Recently, with the report of a precise bottom-up fabrication technique [9], it was possible to synthesize GNR in an easier and more controlled way when compared to other methods, such as chemical vapor deposition [10] and unzipping of carbon nanotubes [43. This new method allows the synthesis of not just rectangular structures but also different GNR shapes called graphene or graphitic nanowiggles (GNW) [9, 14. It uses different types of monomers as molecular precursors in a surface-assisted coupling method [21, 19]. The resulting GNW shape depends on the structure of the precursor monomer, which is easy to control. This enables the experimental synthesis and systematic study of GNW of different shapes.

Basically, GNW consist of non-aligned periodic repetitions of GNR, in a chevron-type graphene nanoribbon structure, as shown in figure 1. The full description of the shape of a GNW depends on four structural parameters [3]: the width of the structure, $L_{o}$ (measured perpendicular to its length) and the length of the oblique, $O_{\beta}$, and outer/inner parallel, $P_{\alpha} / L_{p}$, segments. Oblique and parallel directions are defined with respect to the length direction of the structure (i.e., its longest direction). These parameters are illustrated in Fig. 1(a). The $\alpha / \beta$ sub-index denotes the morphology of the parallel/oblique segment, either armchair $(A)$ or zig-zag $(Z)$. Under this representation, four different GNW families may be defined: $(\alpha, \beta)=(A, A)$ (Fig. 1(a)), $(Z, A)$ (Fig. 1(b)), $(A, Z)$ (Fig. 1(c)) and $(Z, Z)$ (Fig. 1(d)).

It was recently shown that GNW present electronic and magnetic properties that can be tuned just by changing their shape [14]. This enables the tailoring of these structures for specific applications. Although their electronic and spintronic properties have been studied in detail, the study of their mechanical properties and fracture patterns under strain is still missing.

The detailed knowledge of the mechanical properties of these materials is very important for the fabrication of nanodevices and for the exploita- 



Figure 1: Examples of GNW structures. (a) the main four structural parameters $\left(P_{\alpha}, O_{\beta}, L_{p}\right.$ and $\left.L_{o}\right)$ used to uniquely define the GNW structures: $\left(P_{\alpha}\right.$, $\left.O_{\beta}, L_{p}, L_{o}\right)=$ (a) $\left(4_{A}, 8_{A}, 2,7\right)$; (b) $\left(9_{Z}, 15_{A}, 1,17\right)$; (c) $\left(5_{A}, 10_{Z}, 2,9\right)$ and; (d) $\left(20_{Z}, 15_{Z}, 1,39\right)$. In (d) the dotted lines indicate the structural unit cell. For more details about GNW definition and structural characterizations, see reference [13]. 
tion of their graphene-like strength [30, 17]. The mechanical properties of graphene have been intensively investigated by different methodologies, both in experiments and in theory. Based on atomic force microscope (AFM) nanoindentation experiments, it was found that the Young's modulus of free standing graphene sheets could reach values as high as $1.0 \mathrm{TPa}$ and the critical stress (also known as tensile strength and as ultimate strength) could reach $130 \mathrm{GPa}$ [24], which makes graphene the strongest material ever measured. Computer simulations using ab-initio calculations through density functional theory (DFT) are consistent with these results, obtaining 1.05 TPa for the Young's Modulus and $130 \mathrm{GPa}$ for the critical stress [26]. More recently, it was shown that the GNR mechanical properties depend on their width [17, 39, 7], and that they can be harder than graphene and graphene nanotubes due to edge reconstruction effects [17].

However, these remarkable mechanical properties are very sensitive to defects. Even in small amounts they significantly decrease the Young's modulus and the ultimate strength values [1]. In the case of grain boundaries, the mechanical properties remain almost unchanged from the pristine graphene sheet [25], in agreement with previous theoretical predictions [20]. However, when vacancies and Stone-Wales defects are considered, the Young's modulus and ultimate strength decrease with the increase in the density of defects, reaching a saturation point in the high-ratio regime [44]. Additionally, the insertion of defects in graphene leads to changes in their fracture patterns, transitioning from brittle to super-ductile behavior [44].

GNW represent an attempt to tune the mechanical properties of graphenelike materials using structures that do not exhibit vacancies and/or StoneWalles-like defects, although they can be considered themselves as a kind of graphene with topological defects.

In this work, we present a thorough and systematic investigation of the GNW mechanical properties and fracture dynamics. A large number of distinct GNW shapes and sizes were investigated, comprising all four different families. Using reactive molecular dynamics simulations we calculate the Young's modulus, the ultimate strength, the von Mises stress distribution and the fracture patterns for over 1000 unique structures. Two different temperatures, $10 \mathrm{~K}$ and $300 \mathrm{~K}$, were used in order to investigate their temperature dependence. Our results show that the GNW mechanical response can be tuned to a large range of values, while being very sensitive to $P_{\alpha}$, $O_{\beta}, L_{p}$ and $L_{o}$ values. This tunable mechanical behaviour associated with tunable electronic and magnetic properties [14, makes GNW very attractive structures to be exploited as advanced functional materials. 


\section{Methodology}

The present study was carried out through molecular dynamics (MD) simulations using the reactive force field ReaxFF [40, as implemented in the LAMMPS package [32]. Simulations were performed using an accurate timestep of $0.05 \mathrm{fs}$ at two different temperatures, $10 \mathrm{~K}$ and $300 \mathrm{~K}$. The temperature values were controlled by a chain of Nosé-Hoover thermostats. The results discussed below are for $10 \mathrm{~K}$ unless otherwise stated. Results for $300 \mathrm{~K}$ will be discussed when relevant.

ReaxFF is a general distance-dependent bond order potential in which the van der Waals and Coulomb interactions are explicitly considered [35. It can reliably describe the formation and dissociation of chemical bonds among atoms, thus allowing the study of chemical reactions. Its use is attractive in cases where the use of $a b$ initio methods becomes computationally prohibitive, i.e., for large systems and for long simulation times. The force field parametrization was developed using very accurate DFT calculations and experimental data when available [35. ReaxFF has been successfully used in investigations of mechanical properties of silicene membranes [4], graphene-like carbon nitride sheets [15], graphene healing mechanisms [5], combustion [42, 33] and oxidation [12] of carbon-based systems, etc. In our simulations we adopted Mueller's parametrization [27]. This parametrization has been shown to produce good results in the study of mechanical properties of carbon-based nanostructures.

GNW structures were built with an average of 2000 atoms. The methods for obtaining GNW unit cells are described in reference [13]. Herein, $P_{\alpha}$ and $O_{\beta}$ are measured as the number of lines of carbon atoms parallel to the respective direction and into the corresponding region. These lines are shown in 1(a) for both directions. This is equivalent to measuring them as $2 n+1$, where $n$ is the number of rings along the perpendicular direction, and $n$ can assume semi-integer values. $L_{o}$ is measured similarly to $P_{\alpha}$, however, it accounts for all lines of carbon atoms that are parallel to the GNW length, including the ones within the oblique region. The same formula using the number of rings applies to $L_{o} . L_{p}$ is measured as the number of carbon atoms in the innermost parallel segment that have only 2 nearest-neighbors. For all structures the $L_{p}$ parameter was taken as the smallest possible, i.e., $L_{p}=2$ if $\alpha=A$ or $L_{p}=1$, if $\alpha=Z$. The $L_{o}$ parameter was chosen such that $L_{o}=2 \times P_{\alpha}-1$. In this way, the structures can be defined just by the $P_{\alpha}$ and $O_{\beta}$ parameters. We excluded the forbidden (in terms of carbon valence) geometries of the combination of $P_{\alpha}, O_{\beta}, L_{p}$ and $L_{o}$.

In order to perform stress/strain calculations, we first carefully thermalized the structures using a NPT ensemble, fixing the external pressure to 
zero along the periodic directions (see Fig. 1). The thermalization procedure is performed in order to eliminate any residual stress from thermal effects. After this, we used a NVT ensemble and continuously (until mechanical failure) stretched the structure by applying strain along the periodic directions. Stress values were computed at each time step. We adopted a strain rate of $1 \times 10^{-5} \mathrm{fs}^{-1}$, which was found to be adequate after several tests.

In order to obtain the stress values, we calculated the virial stress tensor, given by

$$
\sigma_{i j}=\frac{\sum_{k}^{N} m_{k} v_{k_{i}} v_{k_{j}}}{V}+\frac{\sum_{k}^{N} r_{k_{i}} \cdot f_{k_{j}}}{V},
$$

in which $V=l \times A$ is the structure volume, $N$ the number of atoms, $v$ the velocity, $r$ the atom position and $f$ the force per atom. The GNW volume was calculated during the stretching process and the total area at zero strain $\left(A_{0}\right)$. $A_{0}$ was calculated by multiplying the total length and the total width of the GNW and then subtracting the area of the trapezoidal regions that are empty. The total area was assumed to grow linearly with the strain, i. e., $A=(1+\epsilon) A_{0}$, where $\epsilon$ is the strain. We adopted the thickness of a graphene sheet as being $l=3.4 \AA$.

The stress-strain curves were obtained by plotting the uniaxial component of the stress tensor $\left(\sigma_{i i}\right)$ along the periodic direction $(i)$ and the strain $\left(\epsilon_{i}\right)$, which is defined as a dimensionless quantity dividing the actual deformation by the initial size of the structure along that direction, i. e.:

$$
\epsilon_{i}=\frac{\Delta L_{i}}{L_{i}^{o}}
$$

where $\Delta L_{i}=L_{i}-L_{i}^{o}$ is the variation along the $i$ direction, $L_{i}$ is the actual dimension and $L_{i}^{o}$ is the initial length of the structure. The Young's modulus values can be obtained as the ratio between the uniaxial stress and the strain applied along the periodic direction at the linear regime

$$
Y=\frac{\sigma_{i i}}{\epsilon_{i}}
$$

where $\sigma_{i i}$ is the $i i$ component of the virial stress tensor.

We also calculated the von Mises stress values for each atom in order to obtain information regarding the stress distribution on the strained structure. The von Mises stress provides helpful information on the fracture process, since it is possible to easily visualize its distribution throughout the whole structure [16, 15, 4]. The von Mises per atom (i) stress is defined as

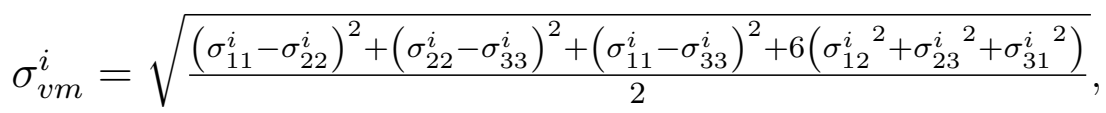


in which the $\sigma_{i i}(i=1,2,3)$ and $\sigma_{i j}(i \neq j=1,2,3)$ components are the normal and shear stresses, respectively.

\section{Results and Discussions}

Firstly, we analyzed the relaxed GNW structures at finite temperatures. These structures are obtained through a thermalization process, as discussed in the methodology section. After these processes, GNW exhibit structural corrugations, as illustrated in Fig. 1 of the supplementary material. The level of corrugation depends on the GNW family ( $A A, A Z, Z A$ or $Z Z$ ) and on the values of $P_{\alpha}$ and $O_{\beta}$. The $A A$ family presents the smallest levels of corrugation, assuming considerable values only for structures with high $P_{\alpha}$ values. For the $A Z$ family, the corrugation levels can be considerable for large values of $P_{\alpha}$ and small values of $O_{\beta}$, but is very small for small values of $P_{\alpha}$. Considering the $Z A$ family, the corrugation level increases for large values of $O_{\beta}$. Finally, for the $Z Z$ family, the corrugation level becomes small only for structures with very small values of $O_{\beta}$ and $P_{\alpha}$, assuming significant levels otherwise. These trends can be better visualized in Fig. 2 of the supplementary material, where we present the average quadratic out-of-plane position $\left.\left(<z^{2}\right\rangle\right)$ for the different structures. As expected, the temperature of the thermalization process also influences the GNW corrugation levels.

We then proceed with the analyses of the GNW mechanical properties under strain. As the strain increases, all levels of corrugations start to decrease, disappearing when the stress begins to increase in a linear regime. From this point, stress begins to increase linearly, characterizing the elastic behaviour of the material. From this linear regime we can calculate the Young's modulus values. In this regime, there is no plastic deformation, i.e., the structure returns to its initial configuration if the strain is removed. As strain continues to be increase, stress increases and the structure presents a non-linear behaviour until it reaches the ultimate strength point $\sigma_{c}$. At this point, the fracture process starts to occur and the stress values fast drops to zero. Representative stress-strain curves for one structure of each family are shown in Fig. 2 (a), where each regime can be easily identified.

During the stretching process, we observe that the von Mises stress accumulates in a central line along the longitudinal direction of the GNW and reaches its maximum value at the inner corners of the structure, as can be seen in Fig. 3. This can be explained by the fact that the regions far from this central line can easily relieve stress due to their unconstrained boundaries. Only the central line is constrained on both sides along the direction of the applied strain. The inner corners accumulate even more stress than 


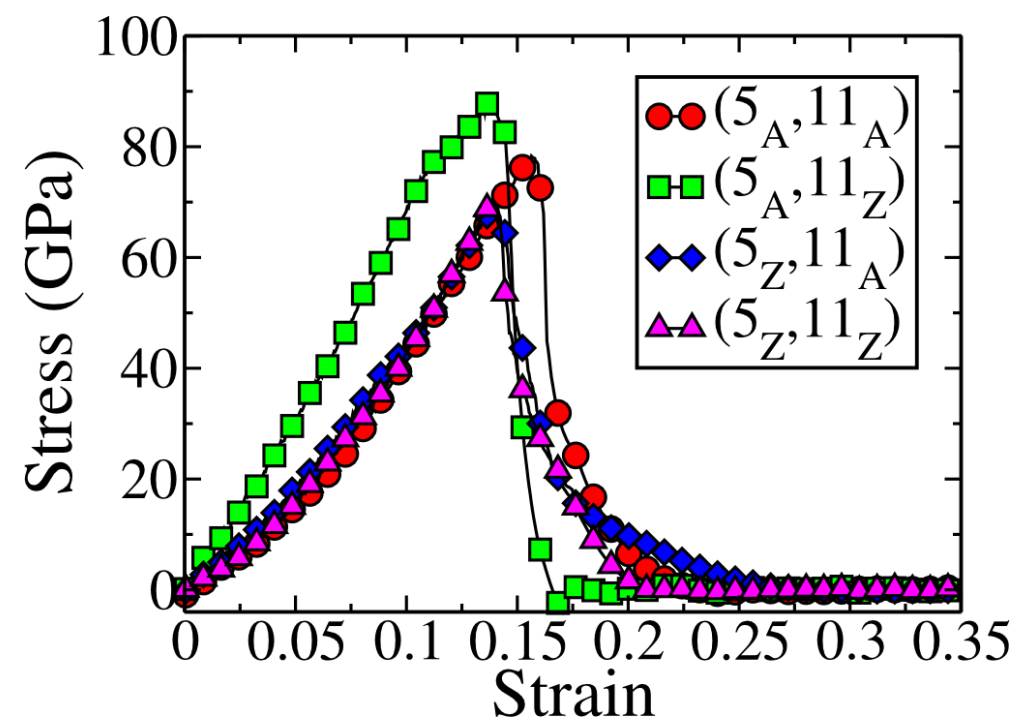

(a)

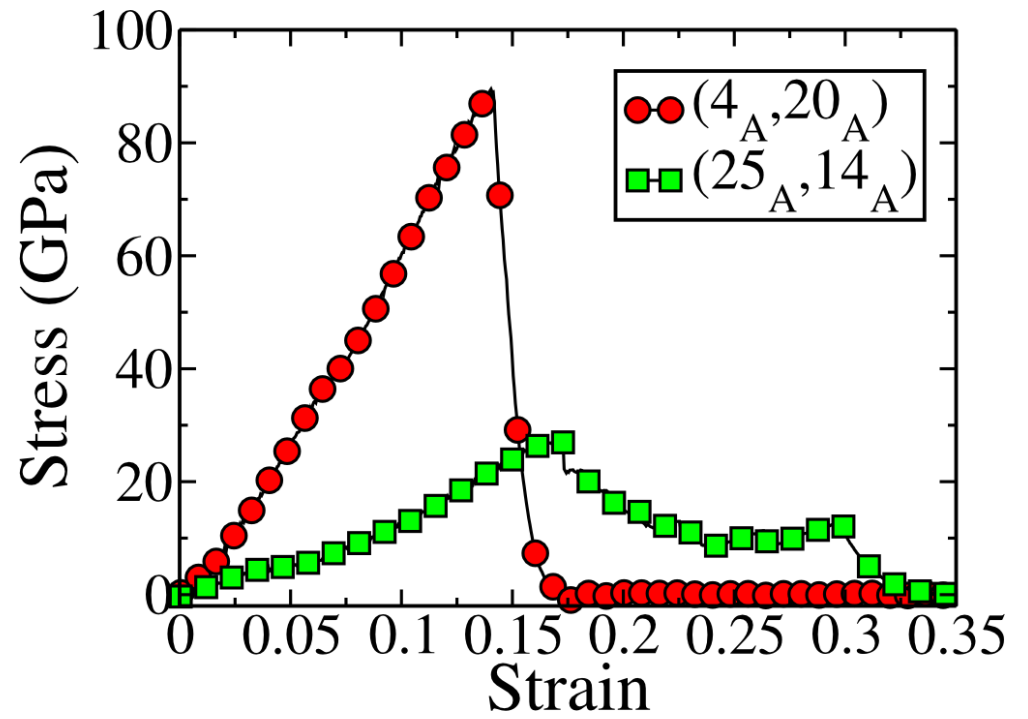

(b)

Figure 2: Representative stress/strain curves. (a) of all GNW families keeping the $P_{\alpha}$ and $O_{\beta}$ indexes constant. The obtained results for Young's modulus $(Y)$ and ultimate strength $\left(\sigma_{c}\right)$ were $Y=648(2) G P a$ and $\sigma_{c} \approx 78 \mathrm{GPa}$ for $\left(5_{A}, 11_{A}\right), Y=763(3) G P a$ and $\sigma_{c} \approx 89$ GPa for $\left(5_{A}, 11_{Z}\right), Y=659(5)$ GPa and $\sigma_{c} \approx 69$ GPa for $\left(5_{Z}, 11_{A}\right)$ and $Y=664(4)$ GPa and $\sigma_{c} \approx 70$ GPa for $\left(5_{Z}, 11_{Z}\right)$; (b) for the AA family showing super-ductility for large $P_{\alpha}$ values. As can be seen, for a $\left(25_{A}, 14_{A}\right) G N W$ fracture behavior is ductile and complete rupture is observed at approximately $30 \%$ strain, while for $\left(4_{A}, 20_{A}\right)$ GNW fracture behavior is brittle and complete rupture is observed at approximately $15 \%$ strain. 

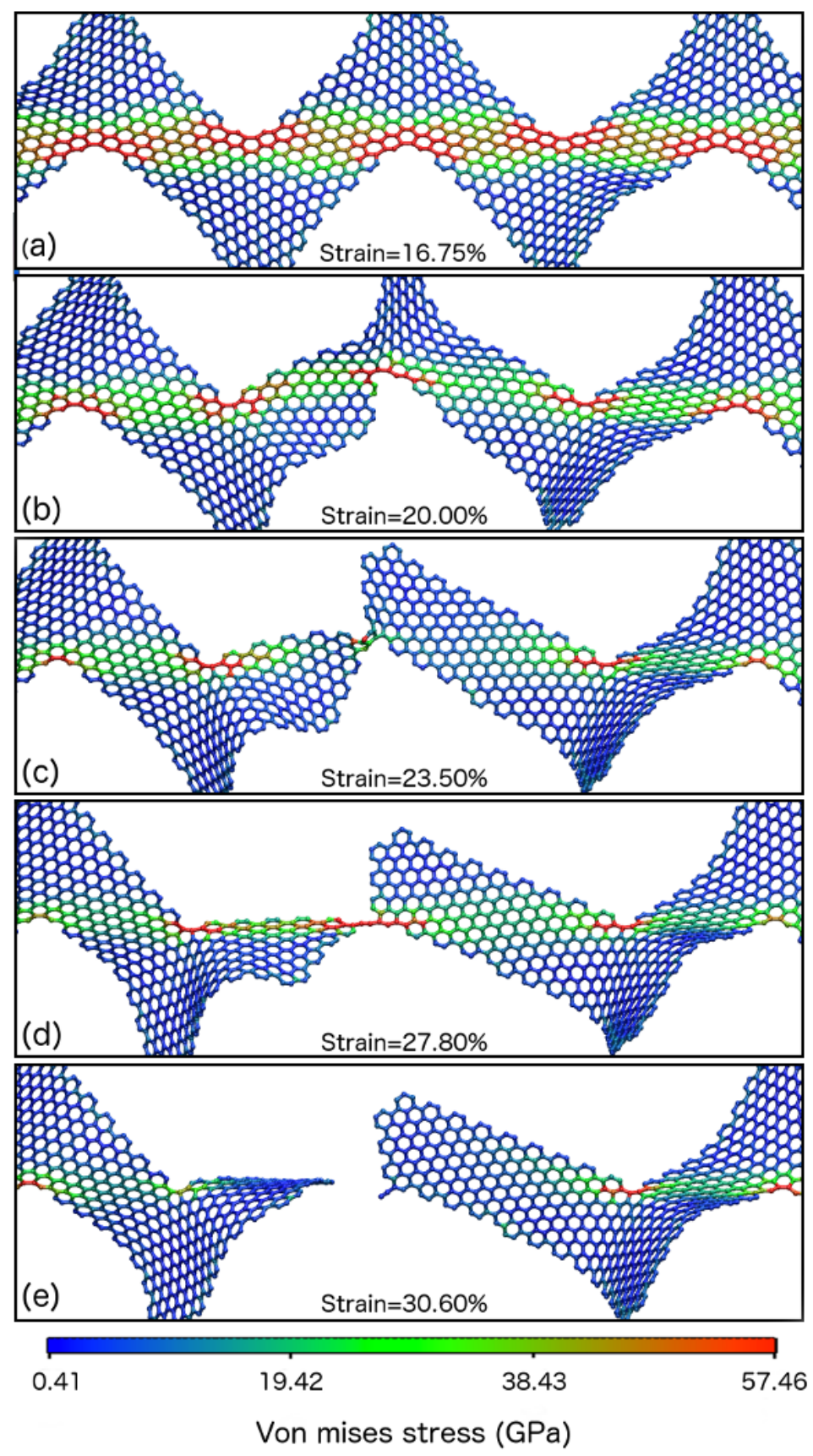

Figure 3: (a-d) Fracture dynamics of the $\left(25_{A}, 14_{A}\right)$ GNW showing the von Mises stress distribution. The stress concentrates on a central line that propagates along the structure. As the strain increases, stress accumulates at the corner region of the structure and increases until mechanical failure (rupture). We have adopted a van der Waals radius $(1.7 \AA)$ for the carbon atom in order to compute the von Mises stress. 


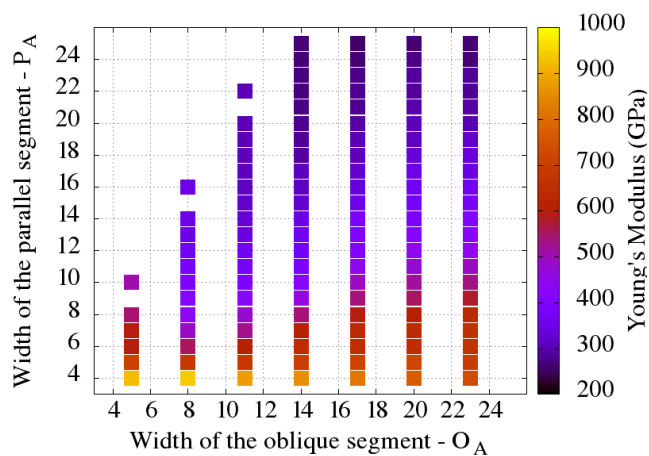

(a)

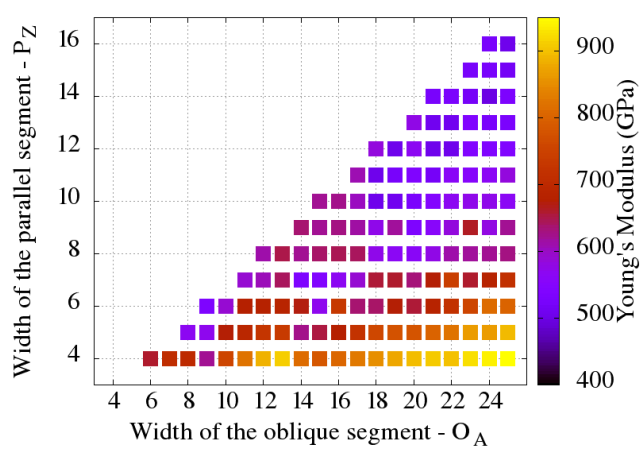

(c)

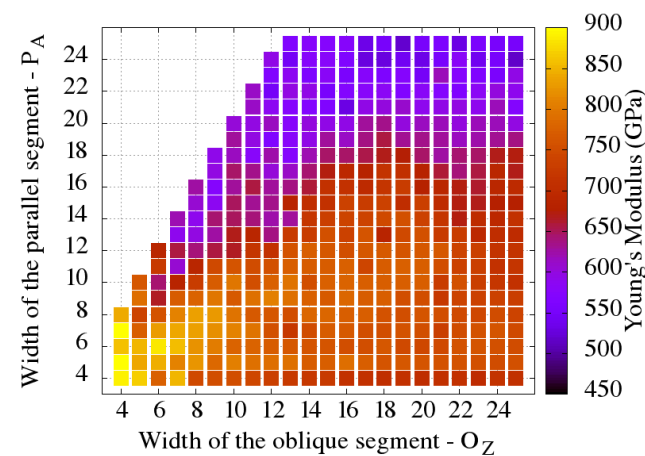

(b)

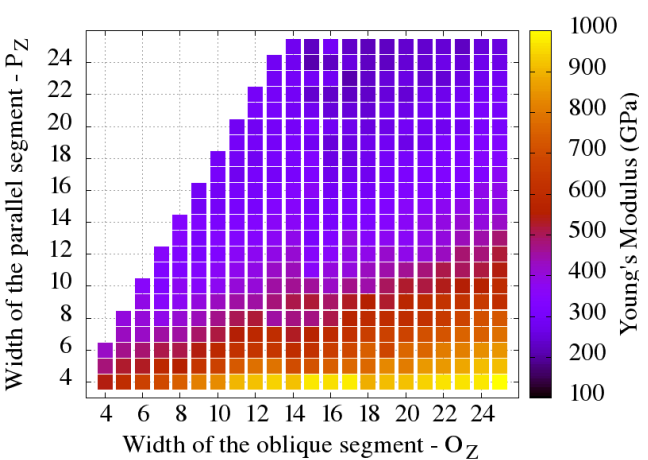

(d)

Figure 4: Dependence of the Young's modulus on the width of the parallel and oblique segments ( $P_{\alpha}$ and $O_{\beta}$, respectively) for (a) $A A$, (b) $A Z$, (c) $Z A$ and (d) ZZ GNW. In general, Young's modulus values decrease with an increase of the parallel segment. 
the rest of the central line because of the force imbalances caused by absence of neighbors in one direction, which eliminates the internal reaction forces that otherwise would distribute the stress.

It is a well-know fact that defects can locally weaken the material, favoring fracture to occur in that region and at lower stress values than the observed for the corresponding pristine material [8, 23. As previously above mentioned, the GNW shape can be considered as topological graphene intrinsic defects, so it should be expected that the GNW ultimate strength should be lower than that of graphene and that the fracture should occur at those regions. That is exactly what we observed in our simulations, since the strongest GNW is still weaker than pristine graphene, with cracks being usually formed at the vertices of the wiggles. This behavior was consistent for all distinct GNW families investigated here.

The nature of the fracture process strongly depends on the shape of the structures, ranging from brittle to super-ductile. A $\left(4_{A}, 20_{A}\right)$ GNW, for example, presents highly brittle behavior, with stress abruptly falling to zero after the fracture starts, as shown in Fig. 2 (b). On the other hand, for a $\left(25_{A}, 14_{A}\right)$ GNW, a ductile behaviour is observed and complete rupture is only obtained for strain values larger than 0.3 . In this case, a more complex process of stress alleviation can be observed, with several successive steps, as shown in Fig. 2 (b), differing from the abrupt decrease observed for graphene [31] and $\left(4_{A}, 20_{A}\right)$ GNW structure. These steps in the stress/strain curve are a consequence of an unravel-like process in the bond breaking that leads to a super-ductile behavior. In general, super-ductile behaviour was observed for structures with large parallel segments, i.e., large $P_{\alpha}$ values. This phenomenon was more pronounced for the $Z Z$ family, with some structures reaching final strain values as high as 0.5 . The final strain values reported here are larger than that ones previously reported by $\mathrm{Xu}$ et al. [44] for graphene with defects, especially when comparing with structures of the $Z Z$ family, as can be seen in Fig. 4 of the supplementary material.

We observed that GNW fractures usually propagate along the oblique directions. In this way, $A A$ and $Z A$ GNW families present armchair edges along the fracture, while $A Z$ GNW family present zigzag edges. On the other hand, an armchair fracture is more common for the $Z Z$ family. Snapshots of the full fracture process of a $\left(25_{A}, 14_{A}\right)$ GNW can be seen in Fig. 3 . The corresponding videos depicting the whole dynamics for $\left(25_{A}, 14_{A}\right)$ and $\left(4_{A}, 20_{A}\right)$ GNW can be found in the supplementary material.

Considering all GNW families, the Young's modulus and the ultimate strength $\sigma_{c}$ values decrease with the increase of the $P_{\alpha}$ parameter. However, we could not verify a consistent dependence on the $O_{\beta}$ parameter. The values of the Young's modulus for all investigated GNW structures are presented 
in Fig. 4. The ultimate strength, $\sigma_{c}$, follows a similar trend, which can be seen in Figs. 3 and 5 of the supplementary material. Temperature effects are negligible on the Young's modulus values (see Fig. 6 of the supplementary material), as they remain virtually unchanged from $10 K$ to $300 K$. On the other hand, ultimate strength values are very sensitive to temperature changes, sharply decreasing with increasing temperature.

Among the four families, the Young's modulus for the $A A$ and $Z Z$ families can reach values from 100 to $1000 \mathrm{GPa}$ (Fig. 4 (a) and (d)), while for the $A Z$ and $Z A$ families the values range from 400 to $900 \mathrm{GPa}$ (Fig. 4 (b) and (c)). The ultimate strength values range from 20 to $100 \mathrm{GPa}$ for $A A, 40$ to 110 for $A Z, 40$ to 90 for $Z A$, and 20 to 100 for $Z Z$. The very large range of Young's modulus, final strain and ultimate strength values provides great tunability to the GNW mechanical properties, enabling them to be tailored for specific applications.

Another interesting result is that the ultimate strength values depend on the $P_{\alpha}$ parameter in the form of a power law, as can be seen in Fig. 5. For this analysis, the $O_{\beta}$ parameter was kept constant at $O_{\beta}=23$. The exponents of the power law depend on the GNW family. The $Z Z$ and $A A$ families present the largest exponents: $\gamma=0.83(1)$ for $Z Z$ family and $\gamma=0.71(2)$ for $A A$ family, followed by the $Z A$ family with $\gamma=0.51(2)$ and $A Z$ family with $\gamma=0.40(2)$. The power law regressions may be very useful to estimate the ultimate strength of uncalculated GNW structures.

The fact that $A Z$ and $Z A$ families have larger ultimate strength may be attributed to their larger opening angle $\left(120^{\circ}\right)$ when compared to $A A$ and $Z Z$ families $\left(60^{\circ}\right)$. As previously attested by Carpinteri [11], structures with re-entrant corners get stronger when the mass of the structure decreases, i.e. the angle of the corner increases.

\section{Conclusions}

We have investigated through fully atomistic reactive molecular dynamics simulations the mechanical properties of graphene (graphitic) nanowiggles under strain for different temperatures. We calculated the Young's modulus, ultimate strength and stress distribution during the stretching process, as well as the fracture patterns for different GNW families.

GNW are shown to present very diverse mechanical properties, which strongly depend on their shape. In special, a super-ductile behavior was observed for structures with large values of $P_{\alpha}$, while brittle behavior was the general trend for the other GNW. The fracture dynamics for the superductile structures present an unravel-like process of the hexagonal rings near 


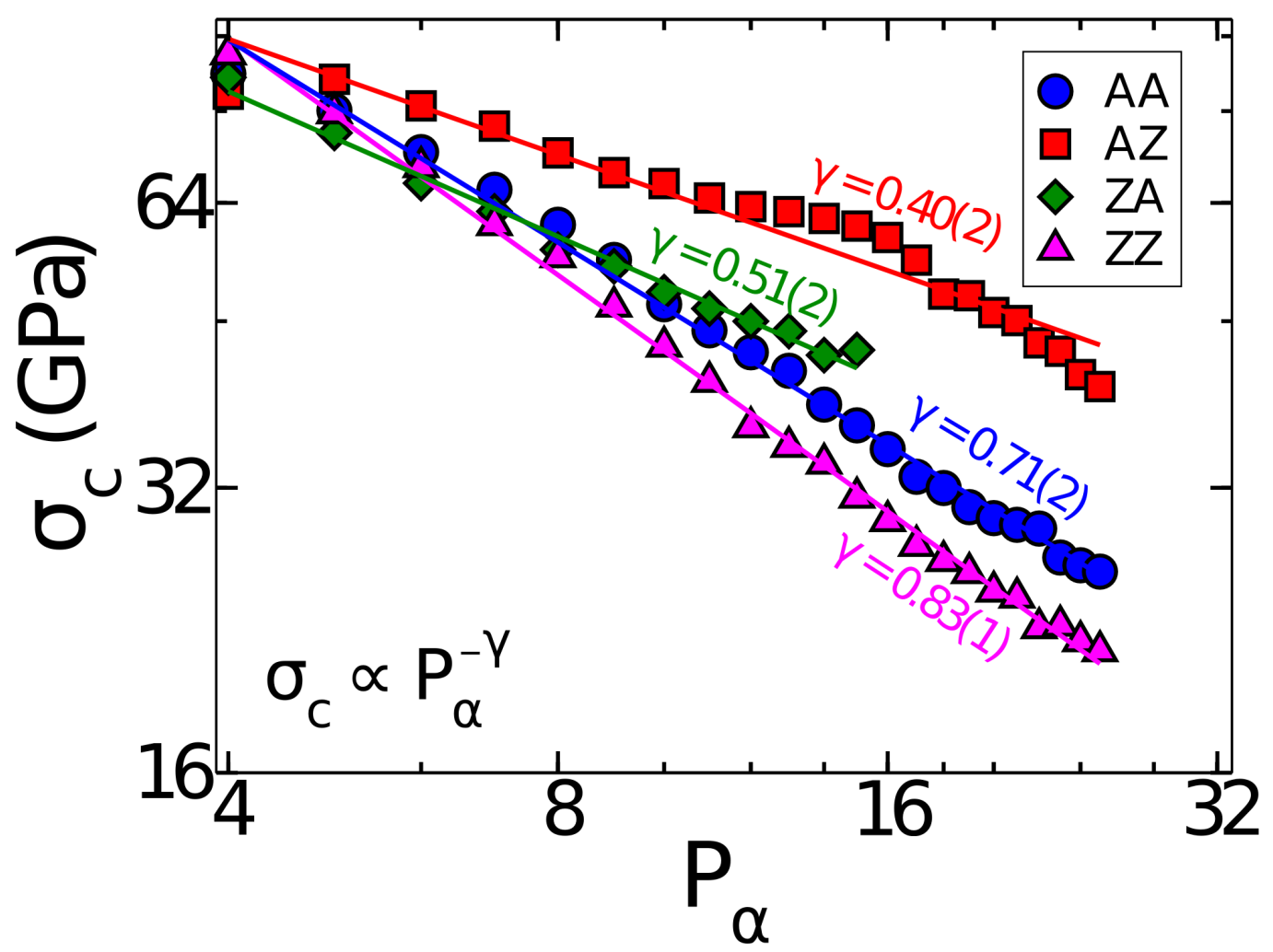

Figure 5: Ultimate strength (US) as a function of $P_{\alpha}$ keeping $O_{\beta}$ parameter constant at $O_{\beta}=23$ for all the GNW families. It can be seen that the ultimate strength follows a power law, decreasing while the $P_{\alpha}$ parameter grows. 
to the inner corners. For some structures, complete fracture happens for strain values as high as 0.5 .

Young's modulus and ultimate strength values range from 100 to 1000 GPa and 20 to $110 \mathrm{GPa}$, respectively. Also, a power law dependence on the width of the parallel segment, $P_{\alpha}$, was found for the ultimate strength. The wide range of values for Young's moduli, final strains and ultimate strengths and the distinct fracture behaviors provide GNW with an unusual and highly promising level of design versatility. The direct dependence of these properties on the shape of the GNW creates an easily accessible path for tuning. Combining this rich mechanical behavior with their previously reported tunable electric and magnetic properties [14, makes GNW one of the most exciting and attractive novel structures to be exploited as the basis for nanodevices and advanced functional materials.

\section{Acknowledgments}

This work was supported in part by the Brazilian Agencies CNPq, CAPES and FAPESP. The authors would like to thank the Center for Computational Engineering and Sciences at Unicamp for financial support through the FAPESP/CEPID Grant 2013/08293 - 7. N.M.P. is supported by the European Research Council PoC 2015 "Silkene" No. 693670, by the European Commission H2020 under the Graphene Flagship Core 1 No. 696656 (WP14 "Polymer Nanocomposites") and under the Fet Proactive "Neurofibres" No. 732344.

\section{References}

[1] Florian Banhart, Jani Kotakoski, and Arkady V Krasheninnikov. Structural defects in graphene. ACS Nano, 5(1):26-41, jan 2011.

[2] Jens Baringhaus, Ming Ruan, Frederik Edler, Antonio Tejeda, Muriel Sicot, An-Ping Li, Zhigang Jiang, Edward H. Conrad, Claire Berger, Christoph Tegenkamp, and Walt A. de Heer. Exceptional ballistic transport in epitaxial graphene nanoribbons. Nature, 506(7488):349-354, February 2014.

[3] Rafael A. Bizao, Tiago Botari, and D. S. Galvao. Mechanical properties of graphene nanowiggles. In MRS Proceedings, volume 2014, pages mrsf13-1658, 2014. 
[4] T. Botari, E. Perim, P. A. S. Autreto, A. C. T. van Duin, R. Paupitz, and D. S. Galvao. Mechanical properties and fracture dynamics of silicene membranes. Phys. Chem. Chem. Phys., 16(36):19417, 2014.

[5] Tiago Botari, Ricardo Paupitz, Pedro Alves da Silva Autreto, and Douglas S. Galvao. Graphene healing mechanisms: A theoretical investigation. Carbon, 99:302 - 309, 2016.

[6] D. W. Boukhvalov and M. I. Katsnelson. Tuning the gap in bilayer graphene using chemical functionalization: Density functional calculations. Phys. Rev. B, 78:085413, Aug 2008.

[7] Hao Bu, Yunfei Chen, Min Zou, Hong Yi, Kedong Bi, and Zhonghua Ni. Atomistic simulations of mechanical properties of graphene nanoribbons. Phys. Lett. A, 373(37):3359-3362, September 2009.

[8] Markus J Buehler. Atomistic modeling of materials failure. Springer, 2008.

[9] Jinming Cai, Pascal Ruffieux, Rached Jaafar, Marco Bieri, Thomas Braun, Stephan Blankenburg, Matthias Muoth, Ari P Seitsonen, Moussa Saleh, Xinliang Feng, Klaus Müllen, and Roman Fasel. Atomically precise bottom-up fabrication of graphene nanoribbons. Nature, 466(7305):470-3, July 2010.

[10] Jessica Campos-Delgado, José Manuel Romo-Herrera, Xiaoting Jia, David a. Cullen, Hiroyuki Muramatsu, Yoong Ahm Kim, Takuya Hayashi, Zhifeng Ren, David J. Smith, Yu Okuno, Tomonori Ohba, Hirofumi Kanoh, Katsumi Kaneko, Morinobu Endo, Humberto Terrones, Mildred S. Dresselhaus, and Mauricio Terrones. Bulk production of a new form of sp 2 carbon: Crystalline graphene nanoribbons. Nano Lett., 8(9):2773-2778, 2008.

[11] Alberto Carpinteri and Nicola Pugno. Fracture instability and limit strength condition in structures with re-entrant corners. Engineering Fracture Mechanics, 72(8):1254 - 1267, 2005.

[12] Kimberly Chenoweth, Adri C T van Duin, and William A Goddard. ReaxFF reactive force field for molecular dynamics simulations of hydrocarbon oxidation. J. Phys. Chem. A, 112(5):1040-53, February 2008.

[13] Eduardo Costa Girão, Eduardo Cruz-Silva, Liangbo Liang, Antônio Gomes Souza Filho, and Vincent Meunier. Structural and electronic 
properties of graphitic nanowiggles. Phys. Rev. B, 85(23):235431, June 2012.

[14] Eduardo Costa Girão, Liangbo Liang, Eduardo Cruz-Silva, Antônio Gomes Souza Filho, and Vincent Meunier. Emergence of Atypical Properties in Assembled Graphene Nanoribbons. Phys. Rev. Lett., 107(13):135501, September 2011.

[15] J. M. de Sousa, T. Botari, E. Perim, R. A. Bizao, and Douglas S. Galvao. Mechanical and structural properties of graphene-like carbon nitride sheets. RSC Adv., 6:76915-76921, 2016.

[16] R P B dos Santos, E Perim, P A S Autreto, Gustavo Brunetto, and D S Galvao. On the unzipping of multiwalled carbon nanotubes. Nanotechnology, 23(46):465702, 2012.

[17] Ricardo Faccio, Pablo A Denis, Helena Pardo, Cecilia Goyenola, and Alvaro W Mombrú. Mechanical properties of graphene nanoribbons. J. Phys. Condensed Matter, 21(28):285304, July 2009.

[18] A K Geim and K S Novoselov. The rise of graphene. Nature Mater., 6(3):183-91, March 2007.

[19] André Gourdon. On-surface covalent coupling in ultrahigh vacuum. Ang. Chem. Int. Ed., 47(37):6950-6953, 2008.

[20] Rassin Grantab, Vivek B Shenoy, and Rodney S Ruoff. Anomalous Strength Characteristics of Tilt Grain Boundaries in Graphene. Science, 330(6006):946-948, 2010.

[21] Leonhard Grill, Matthew Dyer, Leif Lafferentz, Mats Persson, Maike V Peters, and Stefan Hecht. Nano-architectures by covalent assembly of molecular building blocks. Nature Nano, 2(11):687-691, November 2007.

[22] Melinda Han, Barbaros Özyilmaz, Yuanbo Zhang, and Philip Kim. Energy Band-Gap Engineering of Graphene Nanoribbons. Phys. Rev. Lett., 98(20):206805, May 2007.

[23] Charles Edward Inglis. Stresses in a plate due to the presence of cracks and sharp corners. SPIE MILESTONE SERIES MS, 137:3-17, 1997.

[24] Changgu Lee, Xiaoding Wei, JW Kysar, and James Hone. Measurement of the elastic properties and intrinsic strength of monolayer graphene. Science, 321:385-388, 2008. 
[25] Gwan-Hyoung Lee, Ryan C Cooper, Sung Joo An, Sunwoo Lee, Arend van der Zande, Nicholas Petrone, Alexandra G Hammerberg, Changgu Lee, Bryan Crawford, Warren Oliver, Jeffrey W Kysar, and James Hone. High-strength chemical-vapor-deposited graphene and grain boundaries. Science, 340(May):1073-1076, 2013.

[26] Fang Liu, Pingbing Ming, and Ju Li. Ab initio calculation of ideal strength and phonon instability of graphene under tension. Phys. Rev. $B, 76: 1-7,2007$.

[27] Jonathan E. Mueller, Adri C. T. van Duin, and William A. Goddard. Development and validation of reaxff reactive force field for hydrocarbon chemistry catalyzed by nickel. J. Phys. Chem. C, 114(11):4939-4949, 2010 .

[28] Rahul Mukherjee, Abhay Varghese Thomas, Ajay Krishnamurthy, and Nikhil Koratkar. Photothermally reduced graphene as high-power anodes for lithium-ion Batteries. ACS Nano, 6(9):7867-7878, 2012.

[29] K S Novoselov, A K Geim, S V Morozov, D Jiang, Y Zhang, S V Dubonos, I V Grigorieva, and A A Firsov. Electric field effect in atomically thin carbon films. Science, 306(5696):666-9, October 2004.

[30] IA Ovid'ko. MECHANICAL PROPERTIES OF GRAPHENE. Rev. Adv. Mater. Sci., 34:1-11, 2013.

[31] IA Ovid'ko. Mechanical properties of graphene. Rev. Adv. Mater. Sci, $34: 1-11,2013$.

[32] Steve Plimpton. Fast parallel algorithms for short-range molecular dynamics. J. Comp. Phys., 117(1):1 - 19, 1995.

[33] Hu-Jun Qian, Adri C. T. van Duin, Keiji Morokuma, and Stephan Irle. Reactive Molecular Dynamics Simulation of Fullerene Combustion Synthesis: ReaxFF vs DFTB Potentials. J. Chem. Theor. Comp., 7(7):2040-2048, July 2011.

[34] Marc P Ramuz, Michael Vosgueritchian, Peng Wei, Chenggong Wang, Yongli Gao, Yingpeng Wu, Yongsheng Chen, and Zhenan Bao. Evaluation of Solution-Processable Carbon-Based Electrodes for All-Carbon Solar Cells. ACS Nano, 6(11):10384-10395, 2012. 
[35] Anthony K Rappé, Carla J Casewit, KS Colwell, WA Goddard III, and WM Skiff. Uff, a full periodic table force field for molecular mechanics and molecular dynamics simulations. J. Amer. Chem. Soc., 114(25):10024-10035, 1992.

[36] Frank Schwierz. Graphene transistors. Nature Nano, 5(7):487-496, jul 2010 .

[37] Meryl D Stoller, Sungjin Park, Yanwu Zhu, Jinho An, and Rodney S Ruoff. Graphene-based ultracapacitors. Nano Lett., 8:6-10, 2008.

[38] Mauricio Terrones, Andrés R Botello-Méndez, Jessica Campos-Delgado, Florentino López-Urías, Yadira I Vega-Cantú, Fernando J RodríguezMacías, Ana Laura Elías, Emilio Muñoz Sandoval, Abraham G CanoMárquez, Jean-Christophe Charlier, and Humberto Terrones. Graphene and graphite nanoribbons: Morphology, properties, synthesis, defects and applications. Nano Today, 5(4):351-372, 2010.

[39] Mehmet Topsakal and Salim Ciraci. Elastic and plastic deformation of graphene, silicene, and boron nitride honeycomb nanoribbons under uniaxial tension: A first-principles density-functional theory study. Phys. Rev. B, 81(2):024107, January 2010.

[40] Adri C. T. van Duin, Siddharth Dasgupta, Francois Lorant, and William A. Goddard. ReaxFF: A Reactive Force Field for Hydrocarbons. J.Phys.Chem. A, 105(41):9396-9409, October 2001.

[41] B. Verberck, B. Partoens, F. M. Peeters, and B. Trauzettel. Straininduced band gaps in bilayer graphene. Phys. Rev. B, 85:125403, Mar 2012 .

[42] Michael R Weismiller, Adri C T van Duin, Jongguen Lee, and Richard a Yetter. ReaxFF reactive force field development and applications for molecular dynamics simulations of ammonia borane dehydrogenation and combustion. J. Phys. Chem. A, 114(17):5485-92, May 2010.

[43] Liming Xie, Hailiang Wang, Chuanhong Jin, Xinran Wang, Liying Jiao, Kazu Suenaga, and Hongjie Dai. Graphene Nanoribbons from Unzipped Carbon Nanotubes: Atomic Structures, Raman Spectroscopy, and Electrical Properties. J. Amer. Chem. Soc., 133(27):10394-10397, 2011.

[44] Lanqing Xu, Ning Wei, and Yongping Zheng. Mechanical properties of highly defective graphene: from brittle rupture to ductile fracture. Nanotechnology, 24(50):505703, December 2013. 